Stud. Univ. Babeş-Bolyai Math. 65(2020), No. 3, 411-427

DOI: $10.24193 /$ subbmath.2020.3.09

\title{
Some remarks on linear set-valued differential equations
}

Tatyana A. Komleva, Liliya I. Plotnikova, Natalia V. Skripnik and Andrej V. Plotnikov

\begin{abstract}
The article discusses various definitions of the derivative of a set-valued mapping and their properties. Also, a linear set-valued differential equation is considered and the existence of solutions for this equation with Hukuhara derivative, Plotnikov-Skripnik derivative and Bede-Gal derivative is investigated.
\end{abstract}

Mathematics Subject Classification (2010): 34A60, 34A07, 49J53, 54C60.

Keywords: Linear differential equation, set-valued mapping, Hukuhara difference, derivative.

\section{Introduction}

The set-valued differential, integral and discrete-time equations and inclusions are an important part of the theory of set-valued analysis, and they are high-valued for the control theory and its applications, as well as for fuzzy differential equations. They were first studied in 1969 by F.S. de Blasi and F. Iervolino [5]. Later, set-valued differential equations have been studied by many scientists due to their applications in many areas. A lot of results on the theory of set-valued differential, integral and discrete-time equations and inclusions can be found in the following books and articles $[6,10,12,13,14,15,16,17,22,23,24,25,26,27,31,36,30,38,41,42,44]$ and references therein.

In this article first we consider some definitions of the derivative of a set-valued mapping (Hukuhara derivative [11], Plotnikov-Skripnik derivative [32] and Bede-Gal derivative $[1,19,20,46,47])$ and some of their properties. Next, we consider a linear set-valued differential equation with different derivatives that were previously discussed and study the existence of solutions for these equations. 


\section{Preliminaries}

Let $R$ be the set of real numbers and let $R^{n}$ denote the $n$-dimensional Euclidean space $(n \geq 2)$. We denote by $\operatorname{comp}\left(R^{n}\right)$ and $\operatorname{conv}\left(R^{n}\right)$ the set of nonempty compact subsets of $R^{n}$ and the set of nonempty convex and compact subsets of $R^{n}$, respectively. For two given sets $X, Y \in \operatorname{comp}\left(R^{n}\right)$ and $\lambda \in R$, the Minkowski sum and scalar multiple are defined by

$$
X+Y=\{x+y \mid x \in X, y \in Y\} \quad \text { and } \quad \lambda X=\{\lambda x \mid x \in X\} .
$$

We consider the Hausdorff distance $h: \operatorname{comp}\left(R^{n}\right) \times \operatorname{comp}\left(R^{n}\right) \rightarrow R_{+} \bigcup\{0\}$ given by

$$
h(X, Y)=\min \left\{r \geq 0 \mid X \subset Y+B_{r}(0), Y \subset X+B_{r}(0)\right\},
$$

where $B_{r}(0)=\left\{x \in R^{n} \mid\|x\| \leq r\right\}$ is the closed ball with radius $r$ centered at the origin ( $\|x\|$ denotes the Euclidean norm).

Lemma 2.1. [39, 40] The following properties hold:

1) $\left(\operatorname{conv}\left(R^{n}\right), h\right)$ is a complete metric space,

2) $h(A+C, B+C)=h(A, B)$,

3) $h(\lambda A, \lambda B)=|\lambda| h(A, B)$ for all $A, B, C \in \operatorname{conv}\left(R^{n}\right)$ and $\lambda \in R$.

However, $\operatorname{comp}\left(R^{n}\right)$ and $\operatorname{conv}\left(R^{n}\right)$ are not linear spaces since they do not contain inverse elements for the addition, and therefore difference is not well defined, i.e. if $A \in \operatorname{comp}\left(R^{n}\right)$ and $A \neq\{a\}$, then $A+(-1) A \neq\{0\}$. As a consequence, alternative formulations for difference have been suggested [7, 11, 28, 39]. One of these alternatives is the Hukuhara difference [11].

Definition 2.2. [11] Let $X, Y \in \operatorname{conv}\left(R^{n}\right)$. A set $Z \in \operatorname{conv}\left(R^{n}\right)$ such that $X=Y+Z$ is called a Hukuhara difference (H-difference) of the sets $X$ and $Y$ and is denoted by $X \stackrel{H}{ } Y$.

In this case $X \underline{H}+X=\{0\}$ and also $(A+B) \underline{H} B=A$ for any $A, B \in \operatorname{conv}\left(R^{n}\right)$. Also, we note that $X \underline{H} Y \neq X+(-1) Y$.

Remark 2.3. Let $A, B \in \operatorname{conv}\left(R^{n}\right)$. Then the following statements are true:

1) if the H-difference $A \stackrel{H}{ } B$ exists, then $\operatorname{diam}(A) \geq \operatorname{diam}(B)$;

2) if $n=1$ and $\operatorname{diam}(A) \geq \operatorname{diam}(B)$, then the H-difference $A \underline{H} B$ exists;

3) if $n \geq 2$ and $\operatorname{diam}(A) \geq \operatorname{diam}(B)$, then the H-difference $A \underline{H} B$ may not exist. For example, if $A=\left\{a \in R^{n}|| a_{i} \mid \leq 2, i=\overline{1, n}\right\}$ and $B=\left\{b \in R^{n} \mid\|b\| \leq 1\right\}$, then $A \stackrel{H}{ } B$ does not exist.

The properties of this difference are studied in detail in $[11,15,16,22,31,30,39]$.

M. Hukuhara introduced the concept of H-differentiability [11] for set-valued functions by using the H-difference.

Let $X:[0, T] \rightarrow \operatorname{conv}\left(R^{n}\right)$ be a set-valued mapping; $\left(t_{0}-\Delta, t_{0}+\Delta\right) \subset[0, T]$ be a $\Delta$ - neighborhood of a point $t_{0} \in[0, T] ; \Delta>0$.

For any $t \in\left(t_{0}-\Delta, t_{0}+\Delta\right)$ consider the following Hukuhara differences $X(t) \stackrel{h}{ } X\left(t_{0}\right), t \geq t_{0}$, and $X\left(t_{0}\right) \underline{h} X(t), t \geq t_{0}$ if these differences exist. 
Definition 2.4. [11] We say that the mapping $X:[0, T] \rightarrow \operatorname{conv}\left(R^{n}\right)$ has Hukuhara derivative (H-derivative) $D_{H} X\left(t_{0}\right)$ at a point $t_{0} \in[0, T]$, if there exists an element $D_{H} X\left(t_{0}\right) \in \operatorname{conv}\left(R^{n}\right)$ such that the limits

$$
\lim _{t \downarrow t_{0}} \frac{1}{t-t_{0}}\left(X(t) \frac{h}{X} X\left(t_{0}\right)\right) \text { and } \lim _{t \uparrow t_{0}} \frac{1}{t_{0}-t}\left(X\left(t_{0}\right) \frac{h}{X} X(t)\right)
$$

exist in the topology of $\operatorname{conv}\left(R^{n}\right)$ and are equal to $D_{H} X\left(t_{0}\right)$.

The properties of Hukuhara derivative are studied in detail in $[8,11,15,22,31$, 30, 39]. Here, we mention some of them.

Theorem 2.5. [11] If the mapping $X:[0, T] \rightarrow \operatorname{conv}\left(R^{n}\right)$ is H-differentiable on $[0, T]$, then

$$
X(t)=X(0)+\int_{0}^{t} D_{H} X(s) d s,
$$

where the integral is understood in the sense of [11].

Corollary 2.6. If the mapping $X(\cdot)$ is H-differentiable on $[0, T]$, then diam $(X(\cdot))$ is a non-decreasing function on $[0, T]$.

Remark 2.7. The inverse statement is not true. For, example. Let $X(\cdot):[0,1] \rightarrow$ $\operatorname{conv}\left(R^{2}\right)$ be such that $X(t)=A(t) C(t)$, where $A(t)=\left(\begin{array}{cc}\cos (t) & -\sin (t) \\ \sin (t) & \cos (t)\end{array}\right)$ is a rotation matrix, $C(t)=\left\{x \in R^{2}|| x_{i} \mid \leq t, i=1,2\right\}$ is square. Obviously, $\operatorname{diam}(X(t))=\sqrt{2} t$. However, the mapping $X(\cdot)$ is not H-differentiable on $[0,1]$.

Corollary 2.8. If the function $\operatorname{diam}(X(\cdot))$ is a decreasing function on $[0, T]$, then the mapping $X(\cdot)$ is not $H$-differentiable on $[0, T]$.

In order to overcome these shortcomings of this approach, other types of derivatives for set-valued functions have been explored.

The first alternative of the derivative for set-valued mappings have been introduced by H.T. Banks, M.Q. Jacobs [7] and J.N.Tyurin [45]. According to the Radströms embedding theorem [40] there is a real normed linear space $\mathcal{B}$ and an isometric mapping $\pi: \operatorname{conv}\left(R^{n}\right) \rightarrow \mathcal{B}$. $\mathcal{B}$ is a space of equivalence classes (see $\left.[7,39,40]\right)$. Then, taking advantage of this embedding theorem, a set-valued mapping $X(\cdot)$ is said to be $\pi$-differentiable at $t_{0}$ if $\pi \circ X(\cdot)$ is differentiable at $t_{0}$. Some properties of this derivative and its connection with other derivatives for set-valued mappings can be found in $[7,9,18,21,37,39]$. However, the $\pi$-derivative of a set-valued mapping $X(\cdot)$ may be an element of the space $\mathcal{B}$, which does not have a comparable set in the space $\operatorname{conv}\left(R^{n}\right)$ (examples, see $\left.[15,22,31,30]\right)$.

In $[28,31,30]$ the definition of the $\mathrm{T}$-derivative that generalizes the $\mathrm{H}$-derivative and reminds outwardly the $\pi$ - derivative was introduced. However, its use had difficulty when writing the corresponding set-valued differential equation.

Later, A.V. Plotnikov and N.V. Skripnik took advantage of some approaches that were used in [28] and introduced a new definition of a derivative. 
Definition 2.9. [32] Let $X:[0, T] \rightarrow \operatorname{conv}\left(R^{n}\right)$ and $t \in[0, T]$. We say that $X(\cdot)$ has a Plotnikov-Skripnik derivative (PS-derivative) $D_{p s} X(t) \in \operatorname{conv}\left(R^{n}\right)$ at $t \in(0, T)$, if for all $\Delta>0$ that are sufficiently close to 0 , the H-differences and the limits exist in at least one of the following expressions:

(i) $\lim _{\Delta \rightarrow 0} \Delta^{-1}\left(X(t+\Delta) \frac{H}{\text { or }} X(t)\right)=\lim _{\Delta \rightarrow 0} \Delta^{-1}(X(t) \underline{H} X(t-\Delta))=D_{p s} X(t)$

(ii) $\lim _{\Delta \rightarrow 0} \Delta^{-1}\left(X(t) \frac{H}{X} X(t+\Delta)\right)=\lim _{\Delta \rightarrow 0} \Delta^{-1}\left(X(t-\Delta) \frac{H}{X}(t)\right)=D_{p s} X(t)$

(iii) $\lim _{\Delta \rightarrow 0} \Delta^{-1}\left(X(t+\Delta) \frac{H}{X} X(t)\right)=\lim _{\Delta \rightarrow 0} \Delta^{-1}\left(X(t-\Delta) \frac{H}{X}(t)\right)=D_{p s} X(t)$ or

(iv) $\lim _{\Delta \rightarrow 0} \Delta^{-1}(X(t) \underline{H} X(t+\Delta))=\lim _{\Delta \rightarrow 0} \Delta^{-1}(X(t) \underline{H} X(t-\Delta))=D_{p s} X(t)$.

The properties of this derivative were obtained in $[32,33,34,35]$. Here, we mention some of them.

Remark 2.10. If the set-valued mapping $X(\cdot)$ is $\mathrm{H}$-differentiable then it is PSdifferentiable and $D_{p s} X(t)=D_{H} X(t)$.

Remark 2.11. If the set-valued mapping $X(\cdot)$ is PS-differentiable on $I$ and $\operatorname{diam} X(\cdot)$ is a non-decreasing function on $[0, T]$ then the set-valued mapping $X(\cdot)$ is $\mathrm{H}$ differentiable and $D_{p s} X(t)=D_{H} X(t)$.

Remark 2.12. There exist set-valued mappings that are PS-differentiable but not H-differentiable.

Example 2.13. The set-valued mapping $X(t)=B_{|t|}(0)$ is PS-differentiable on $R$ and its PS-derivative $D_{p s} X(t) \equiv B_{1}(0)$. It is obvious that the given set-valued mapping is $\mathrm{H}$-differentiable only on the interval $(0,+\infty)$ and $D_{H} X(t)=B_{1}(0)$. On the interval $(-\infty, 0)$ it is not H-differentiable as its diameter on this interval decreases.

Theorem 2.14. [32] If the mapping $X:[0, T] \rightarrow \operatorname{conv}\left(R^{n}\right)$ is PS-differentiable on $[0, T]$, then for all $t \in[0, T]$

(i) if function diam $(X(t))$ is a non-decreasing function on $[0, T]$, then

$$
X(t)=X(0)+\int_{0}^{t} D_{p s} X(s) d s ;
$$

(ii) if function diam $(X(t))$ is a decreasing function on $[0, T]$, then

$$
X(t)=X(0) \frac{H}{t} \int_{0}^{t} D_{p s} X(s) d s .
$$

Later, M.T. Malinowski [19, 20], H. Vu and L.S. Dong [46], H. Vu and N. Van Hoa [47] and Ş.E. Amrahov, A. Khastan, N. Gasilov and A.G. Fatullayev [1] adapted the concept of the Bede-Gal derivative [3, 4, 10, 43] for interval-valued mappings on set-valued mappings, that is, such that $X:[0, T] \rightarrow \operatorname{conv}\left(R^{n}\right)$, and studied its properties [47]. 
Definition 2.15. $[1,46]$ Let $X:[0, T] \rightarrow \operatorname{conv}\left(R^{n}\right)$ and $t \in[0, T]$. We say that $X(\cdot)$ has a Bede-Gal derivative (BG-derivative) $D_{b g} X(t) \in \operatorname{conv}\left(R^{n}\right)$ at $t \in(0, T)$, if for all $\Delta>0$ that are sufficiently close to 0 , the $\mathrm{H}$-differences and the limits exist in at least one of the following expressions:

(i) $\lim _{\Delta \rightarrow 0} \Delta^{-1}(X(t+\Delta) \underline{H} X(t))=\lim _{\Delta \rightarrow 0} \Delta^{-1}(X(t) \underline{H} X(t-\Delta))=D_{b g} X(t)$

(ii) $\lim _{\Delta \rightarrow 0}(-\Delta)^{-1}(X(t) \underline{H} X(t+\Delta))=\lim _{\Delta \rightarrow 0}(-\Delta)^{-1}(X(t-\Delta) \underline{H} X(t))=D_{b g} X(t)$

(iii) $\lim _{\Delta \rightarrow 0} \Delta^{-1}\left(X(t+\Delta) \frac{H}{} X(t)\right)=\lim _{\Delta \rightarrow 0}(-\Delta)^{-1}\left(X(t-\Delta) \frac{H}{X}(t)\right)=D_{b g} X(t)$ or

(iv) $\lim _{\Delta \rightarrow 0}(-\Delta)^{-1}(X(t) \underline{H} X(t+\Delta))=\lim _{\Delta \rightarrow 0} \Delta^{-1}(X(t) \underline{H} X(t-\Delta))=D_{b g} X(t)$.

Remark 2.16. In the article $[19,20]$ M.T. Malinowski considered set-valued mappings that satisfy condition (ii) and called this derivative a second type Hukuhara derivative.

Remark 2.17. If the set-valued mapping $X(\cdot)$ is H-differentiable on $[0, T]$ it is BGdifferentiable on $[0, T]$ and $D_{b g} X(t)=D_{H} X(t)$.

Remark 2.18. If the set-valued mapping $X(\cdot)$ is BG-differentiable on $[0, T]$ and $\operatorname{diam} X(\cdot)$ is a non-decreasing function on $[0, T]$ then the set-valued mapping $X(\cdot)$ is H-differentiable and $D_{b g} X(t)=D_{H} X(t)$.

Remark 2.19. There exist set-valued mappings that are BG-differentiable but not H-differentiable.

Example 2.20. [1] The set-valued mapping $X(t)=B_{|t|}(0)$ is BG-differentiable on $R$ and its BG-derivative $D_{b g} X(t) \equiv B_{1}(0)$. It is obvious that the given set-valued mapping is H-differentiable only on the interval $(0,+\infty)$ and $D_{H} X(t)=B_{1}(0)$. On the interval $(-\infty, 0)$ it is not H-differentiable as its diameter on this interval decreases.

Theorem 2.21. [1] If the mapping $X:[0, T] \rightarrow \operatorname{conv}\left(R^{n}\right)$ is BG-differentiable on $[0, T]$, then for all $t \in[0, T]$

(i) if function diam $(X(t))$ is a non-decreasing function on $[0, T]$, then

$$
X(t)=X(0)+\int_{0}^{t} D_{b g} X(s) d s ;
$$

(ii) if function diam $(X(t))$ is a decreasing function on $[0, T]$, then

$$
X(t)=X(0) \frac{H}{(-1)} \int_{0}^{t} D_{b g} X(s) d s .
$$

Remark 2.22. By Remarks 2.10 and 2.17, if the set-valued mapping $X(\cdot)$ is $\mathrm{H}$ differentiable on $[0, T]$ then it is BG-differentiable on $[0, T]$ and PS-differentiable on $[0, T]$ as well as $D_{H} X(t)=D_{p s} X(t)=D_{b g} X(t)$.

Remark 2.23. By Remarks 2.13 and 2.20, we see that the set-valued mapping $X(t)=$ $B_{|t|}(0)$ is BG-differentiable on $R$ and PS-differentiable on $R$ as well as $D_{b g} X(t) \equiv$ $D_{p s} X(t) \equiv B_{1}(0)$ for all $t \in R$. 
Remark 2.24. There exist set-valued mappings $X(\cdot)$ such that $D_{b g} X(t) \neq D_{p s} X(t)$ for any $t$.

Example 2.25. Let $X:[0,2] \rightarrow \operatorname{conv}\left(R^{2}\right)$ and $X(t)=B_{|1-t|}(g(t))$, where $g(t)=$ $(t+1, t+1)^{T}$ (see Figure 1$)$.

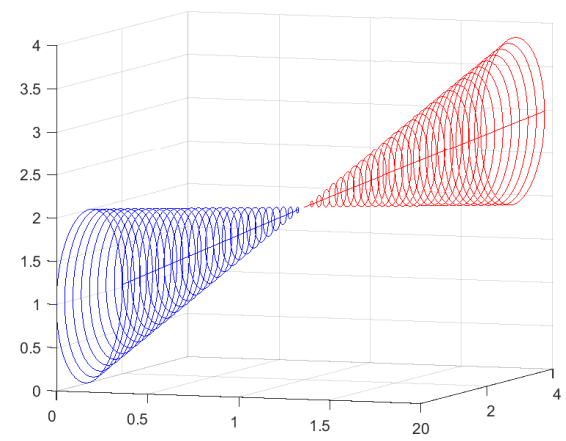

Figure 1: $X(t), t \in[0,2]$

The set-valued mapping $X(\cdot)$ is BG-differentiable on $(0,2)$ and its BG-derivative $D_{b g} X(t) \equiv B_{1}(a)$, where $a=(1,1)^{T}$. However, the set-valued mapping $X(\cdot)$ is PSdifferentiable on $(0,1)$ and its PS-derivative $D_{p s} X(t) \equiv B_{1}(b) \neq D_{b g} X(t)$, where $b=(-1,-1)^{T}$. Also, the set-valued mapping $X(\cdot)$ is PS-differentiable on $(1,2)$ and its PS-derivative $D_{p s} X(t) \equiv B_{1}(a)=D_{b g} X(t)$, where $a=(1,1)^{T}$. As well as the PS-derivative $D_{p s} X(t)$ at the point $t=1$ does not exist (see Figure 2 and Figure 3 ).

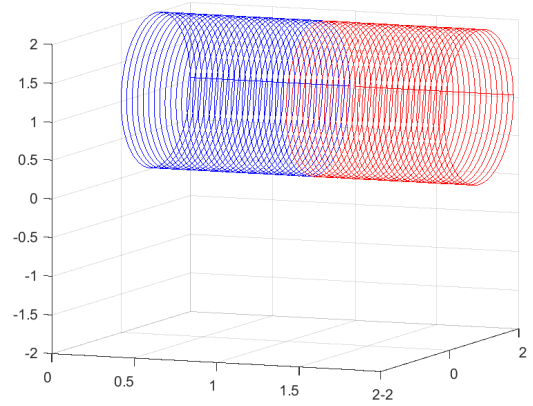

Figure 2: $D_{b g} X(t), t \in[0,2]$

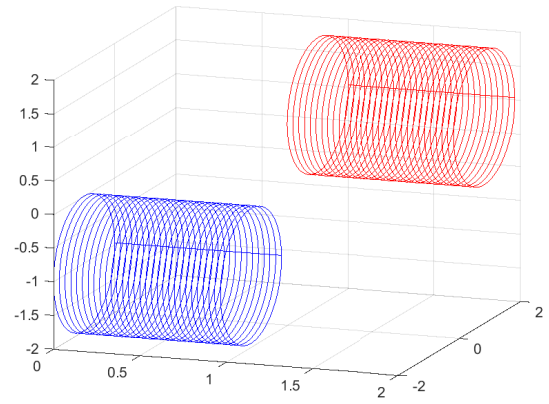

Figure 3: $D_{p s} X(t), t \in[0,2]$

Example 2.26. Let $X:[0,2] \rightarrow \operatorname{conv}\left(R^{2}\right)$ such that

$$
X(t)= \begin{cases}\left\{x \in R^{2} \mid x_{1}^{2}+x_{2}^{2} \leq t, x_{2} \geq 0\right\}, & t \in[0,1], \\ \left\{x \in R^{2} \mid x_{1}^{2}+x_{2}^{2} \leq 2-t, x_{2} \geq 0\right\}, & t \in(1,2]\end{cases}
$$

(see Figure 4). 


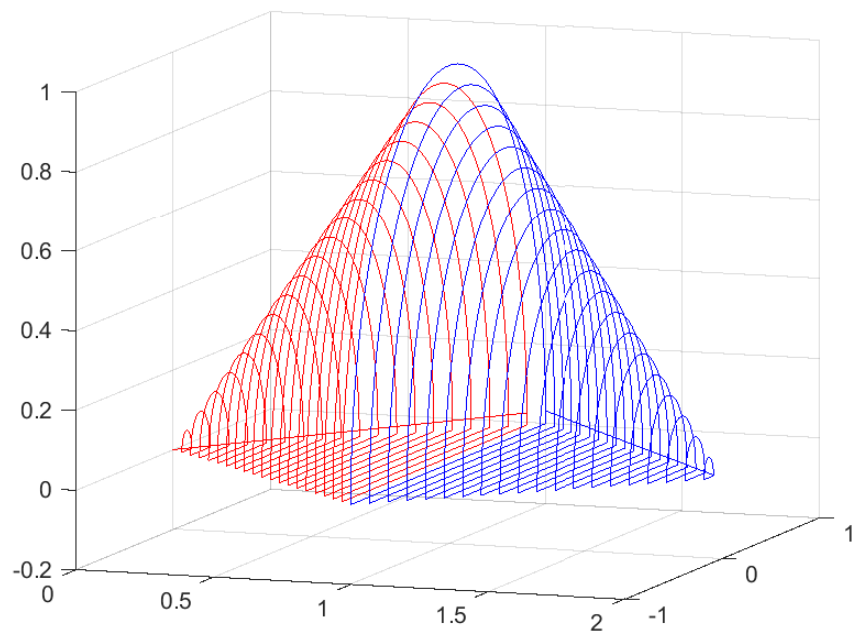

Figure 4: $X(t), t \in[0,2]$

The set-valued mapping $X(\cdot)$ is PS-differentiable on $(0,2)$ and its PS-derivative $D_{p s} X(t) \equiv\left\{x \in R^{2} \mid x_{1}^{2}+x_{2}^{2} \leq 1, x_{2} \geq 0\right\}$. However, the set-valued mapping $X(\cdot)$ is BG-differentiable on $(0,1)$ and its BG-derivative $D_{b g} X(t) \equiv D_{p s} X(t)$. Also, the setvalued mapping $X(\cdot)$ is BG-differentiable on $(1,2)$ and its BG-derivative $D_{b g} X(t) \equiv$ $(-1) D_{p s} X(t)$. As well as the BG-derivative $D_{b g} X(t)$ at the point $t=1$ does not exist (see Figure 5 and Figure 6).

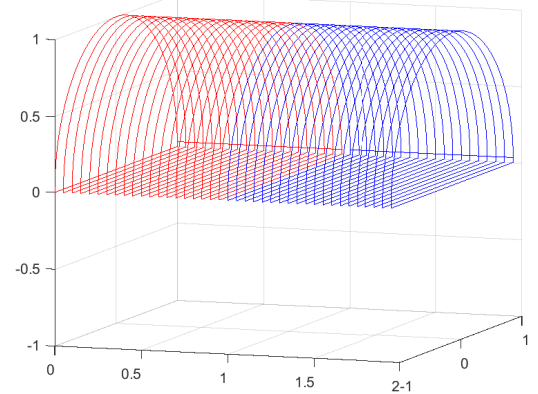

Figure 5: $D_{p s} X(t), t \in[0,2]$

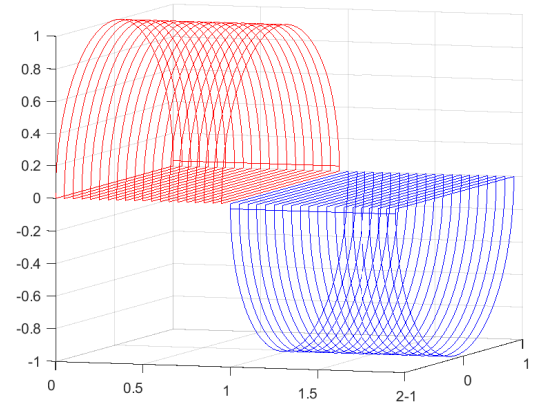

Figure 6: $D_{b g} X(t), t \in[0,2]$

\section{Linear set-valued differential equations}

In this section, we consider linear set-valued differential equations

$$
D X(t)=a X(t), \quad X(0)=X_{0},
$$


where $a \in R, X:[0, T] \rightarrow \operatorname{conv}\left(R^{n}\right)$ is a set-valued mapping, $D X(t)$ is one of the previously considered derivatives $\left(D_{H} X(t), D_{p s} X(t), D_{b g}(t)\right)$ of the set-valued mapping $X(t)$.

Definition 3.1. A set-valued mapping $X(\cdot)$ is called a solution of $(3.1)$ if it is continuously differentiable and satisfies system (3.1) everywhere on $[0, T]$.

As known, linear Hukuhara differential equation

$$
D_{H} X(t)=a X(t), \quad X(0)=X_{0},
$$

has a unique solution on the interval $[0, T][22,31]$. It's also obvious that function $\operatorname{diam}(X(t))$ is a non-decreasing function on $[0, T]$.

Remark 3.2. $[5,22,31]$ If $a \geq 0$ then $X(t)=e^{a t} X_{0}$ for all $t \in[0, T]$.

Remark 3.3. [38] System (3.2) may not be equivalent to the following system of interval-valued differential equations with the Hukuhara derivative

$$
\left\{\begin{array}{rlrl}
D_{H} X_{1}(t) & =a X_{1}(t), & X_{1}(0) & =X_{01}, \\
\cdots & \cdots \\
D_{H} X_{n}(t) & =a X_{n}(t), & X_{n}(0) & =X_{0 n},
\end{array}\right.
$$

where $X_{i}:[0, T] \rightarrow \operatorname{conv}(R)$ is a interval-valued mapping, $X_{0 i}$ is the projection of the set $X_{0}$ on the axis $0 x_{i}, i=\overline{1, n}$.

If $X(\cdot)$ is a solution of $(3.2)$ and $X_{i}(\cdot), i=\overline{1, n}$ are solutions of (3.3), then $X(t) \subset X_{1}(t) \times \ldots \times X_{n}(t)$ for all $t \in[0, T]$.

If $X_{0}=X_{01} \times \ldots \times X_{0 n}$ then system (3.2) is equivalent to system (3.3).

We demonstrate this by the following example.

Example 3.4. Let

$$
D_{H} X(t)=X(t), X(0)=B_{1}(0), t \in[0,1]
$$

and

$$
\begin{cases}D_{H} X_{1}(t)=X_{1}(t), & X_{1}(0)=X_{01}=[-1,1], \\ D_{H} X_{2}(t)=X_{2}(t), & X_{2}(0)=X_{02}=[-1,1],\end{cases}
$$

where $X:[0,1] \rightarrow \operatorname{conv}\left(R^{2}\right)$ is a set-valued mapping, $X_{i}:[0,1] \rightarrow \operatorname{conv}(R)$ is an interval-valued mapping, $X_{0 i}$ is the projection of the set $X_{0}$ on the axis $0 x_{i}, i=\overline{1,2}$.

The set-valued mapping $X(t)=B_{e^{t}}(0)$ is a solution of Hukuhara differential equation (3.4). The interval-valued mappings $X_{i}(t)=\left[-e^{t}, e^{t}\right], i=1,2$ are solutions of the system of Hukuhara differential equations (3.5). It's obvious that $X(t) \subset X_{1}(t) \times$ $X_{2}(t)$ for all $t \in[0,1]$ (see Figure 7). However, if $X(0)=\left\{x \in R^{2}|| x_{i} \mid \leq 1, i=1,2\right\}$ is a square, then $X_{0} \equiv X_{01} \times X_{02}$ and $X(t) \equiv X_{1}(t) \times X_{2}(t)$ for all $t \in[0,1]$ (see Figure 8).

Now, we consider linear differential equation (3.1) with PS-derivative and BGderivative. By $[1,32,33,34,35]$, this set-valued differential equation (3.1) has at least one solution. Moreover, one of these solutions (the one whose diameter is a non-decreasing function) coincides with the solution of the corresponding differential equation (3.2).

We will show it by the following example. 


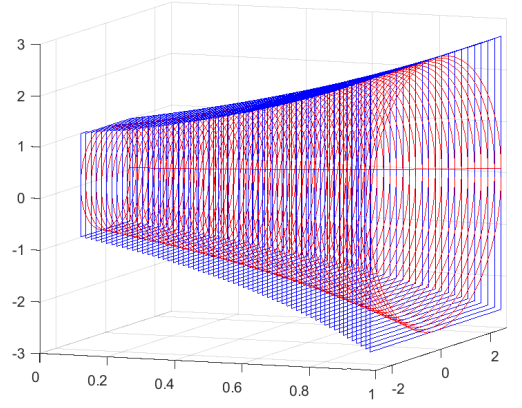

Figure 7:

$$
X(t) \subset X_{1}(t) \times X_{2}(t), t \in[0,1]
$$

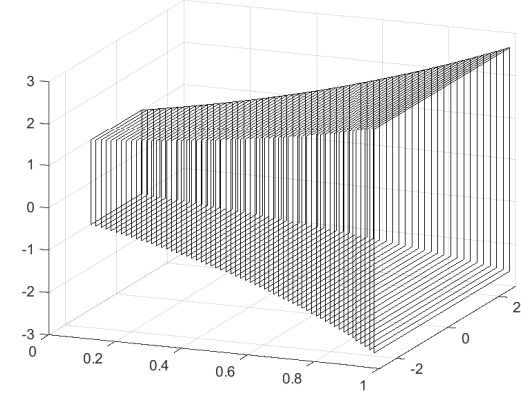

Figure 8:

$$
X(t) \equiv X_{1}(t) \times X_{2}(t), t \in[0,1]
$$

Example 3.5. Let

$$
D X(t)=X(t), X(0)=B_{1}(0), t \in[0,1],
$$

where $X:[0,1] \rightarrow \operatorname{conv}\left(R^{2}\right)$ is a set-valued mapping, $D X(t)$ is one of the previously considered derivatives $\left(D_{H} X(t), D_{p s} X(t), D_{b g}(t)\right)$ of the set-valued mapping $X(t)$.

The set-valued mapping $X(t)=B_{e^{t}}(0)$ is a solution of Hukuhara differential equation (3.6) (see Figure 9).

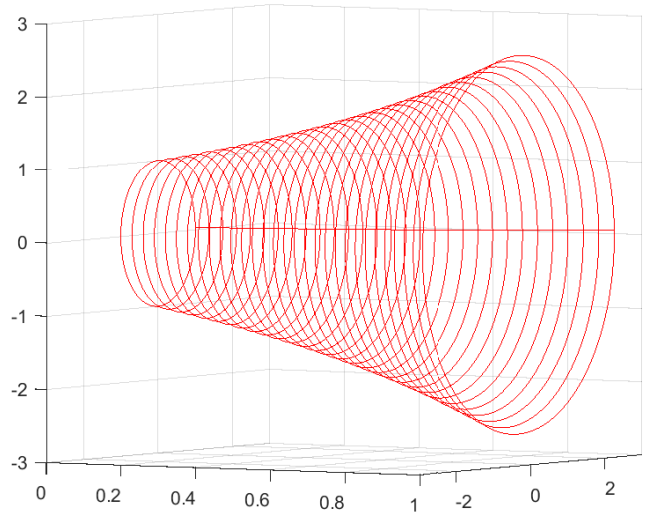

Figure 9: $X(t), t \in[0,1]$

Set-valued mappings $X_{1}(t)=B_{e^{t}}(0)$ and $X_{2}(t)=B_{e^{-t}}(0)$ are solutions of differential equation (3.6) with PS-derivative and BG-derivative (see Figure 10 and Figure 11).

In this case, solutions of differential equations with PS-derivative will be solutions of the differential equation with BG-derivative and vice versa. For the first solution $X_{1}(\cdot)$ the function $\operatorname{diam}\left(X_{1}(t)\right)$ is an increasing function on $[0,1]$. For the second solution $X_{2}(\cdot)$ the function $\operatorname{diam}\left(X_{2}(t)\right)$ is a decreasing function on $[0,1]$. Also, the 


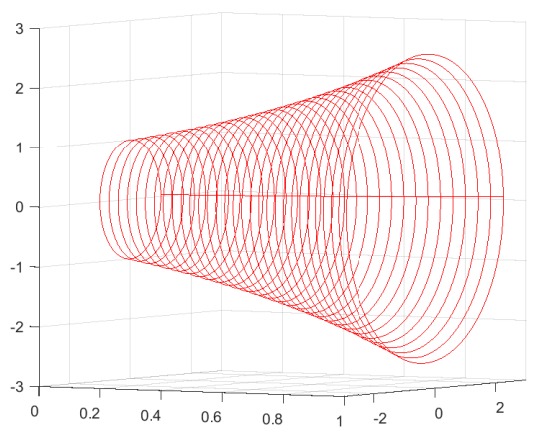

Figure 10: $X_{1}(t), t \in[0,1]$

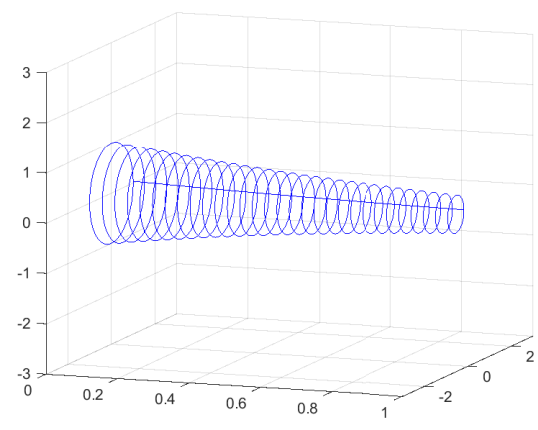

Figure 11: $X_{2}(t), t \in[0,1]$

first solution $X_{1}(\cdot)$ is the solution of the Hukuhara differential equation, i.e. $X(t)=$ $X_{1}(t)$ for all $t \in[0,1]$.

Solutions $X_{1}(\cdot)$ and $X_{2}(\cdot)$ will be called basic solutions.

We also note that set-valued mappings

$$
Y_{1}(t)=\left\{\begin{array}{ll}
B_{e^{t}}(0), & t \in[0,0.5] \\
B_{e^{1-t}}(0), & t \in[0.5,1]
\end{array} \quad Y_{2}(t)= \begin{cases}B_{e^{-t}}(0), & t \in[0,0.5] \\
B_{e^{t-1}}(0), & t \in[0.5,1]\end{cases}\right.
$$

are the solutions of differential equation (3.6) with PS-derivative and BG-derivative (see Figure 12 and Figure 13).

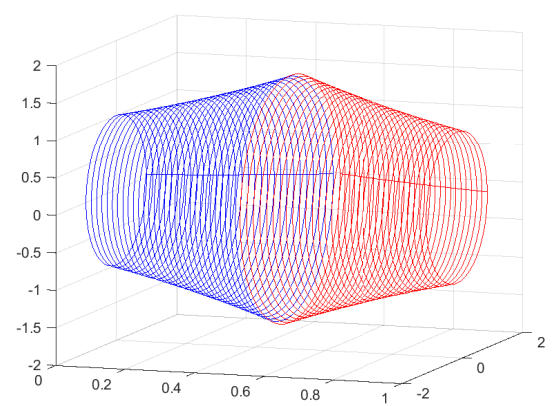

Figure 12: $Y_{1}(t), t \in[0,1]$

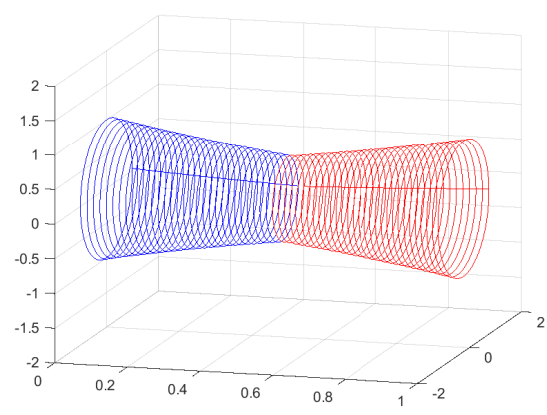

Figure 13: $Y_{2}(t), t \in[0,1]$

It is obvious that in this example such solutions can be built infinitely many. These solutions will be called mixed solutions. For these mixed solutions $Y(\cdot)$, the diameter function $\operatorname{diam}(Y(\cdot))$ is not increasing or decreasing over the entire interval. We also note that the shape of the cross section of solutions corresponds to the shape of the initial set.

Later in this article we will consider only the basic solutions.

The question arises: Do such equations always have two basic solutions?

Consider the following examples when $a=1(a>0)$. 
Example 3.6. Let

$$
D_{p s} X(t)=X(t), X(0)=K, t \in[0,1],
$$

where $X:[0,1] \rightarrow \operatorname{conv}\left(R^{2}\right)$ is a set-valued mapping,

$$
K=\left\{x \in R^{2} \mid x_{1}^{2}+x_{2}^{2} \leq 1, x_{2} \geq 0\right\} .
$$

This differential equation with PS-derivative has two basic solutions $X_{1}(\cdot)$ and $X_{2}(\cdot)$ (see Figure 14 and Figure 15).

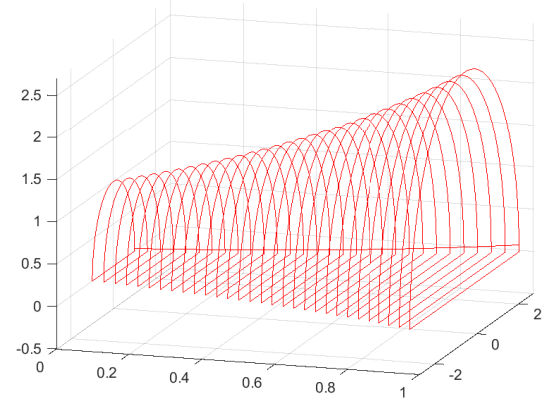

Figure 14: $X_{1}(t), t \in[0,1]$

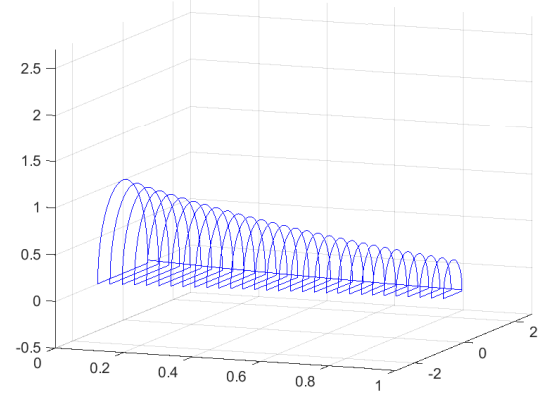

Figure 15: $X_{2}(t), t \in[0,1]$

Example 3.7. Let

$$
D_{b g} X(t)=X(t), X(0)=K, t \in[0,1] .
$$

This differential equation with BG-derivative has only one basic solution, which coincides with the solution of the Hukuhara differential equation and the first basic solution $X_{1}(\cdot)$ of the differential equation with the PS-derivative (see Figure 14).

There will be no second solution because there is no set-valued mapping that satisfies the corresponding integral equation (since the set $K$ is not a centrally symmetric set, the Hukuhara difference does not exist)

$$
X(t)=K \frac{H}{(-1)} \int_{0}^{t} D_{b g} X(s) d s=K \frac{H}{(-1)} \int_{0}^{t} X(s) d s .
$$

Now, we consider the same examples when $a=-1(a<0)$.

Example 3.8. Let

$$
D_{b g} X(t)=(-1) X(t), X(0)=K, t \in[0,1],
$$

where $X:[0,1] \rightarrow \operatorname{conv}\left(R^{2}\right)$ is a set-valued mapping,

$$
K=\left\{x \in R^{2} \mid x_{1}^{2}+x_{2}^{2} \leq 1, x_{2} \geq 0\right\} .
$$

This differential equation with BG-derivative has two basic solutions $X_{1}(\cdot)$ and $X_{2}(\cdot)$ (see Figure 16 and Figure 17). 


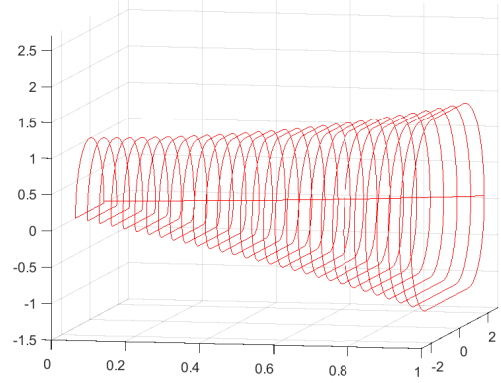

Figure 16: $X_{1}(t), t \in[0,1]$

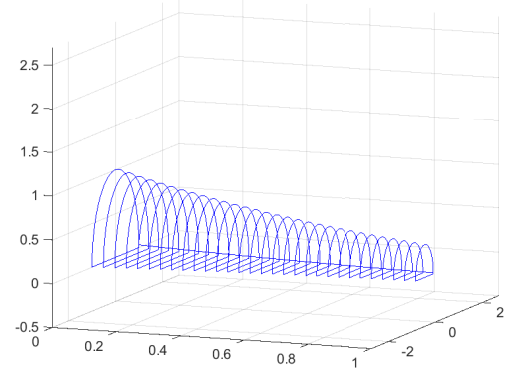

Figure 17: $X_{2}(t), t \in[0,1]$

Example 3.9. Let

$$
D_{p s} X(t)=(-1) X(t), X(0)=K, t \in[0,1] .
$$

This differential equation with PS-derivative has only one basic solution, which coincides with the solution of the Hukuhara differential equation and the first basic solution $X_{1}(\cdot)$ of the differential equation with the BG-derivative.

There will be no second basic solution because there is no set-valued mapping that satisfies the corresponding integral equation (the Hukuhara difference does not exist) $X(t)=K \underline{H} \int_{0}^{t} D_{p s} X(s) d s=K \underline{H}(-1) \int_{0}^{t} X(s) d s$.

Next, we consider the same examples when $X_{0}$ is such that H-difference $X_{0} \underline{H}(-1) X_{0}$ exists $\left(X_{0}\right.$ is centrally symmetric set [7]).

Example 3.10. Let

$$
\begin{aligned}
& D_{b g} X(t)=X(t), X(0)=P, t \in[0,1], \\
& D_{p s} X(t)=X(t), X(0)=P, t \in[0,1],
\end{aligned}
$$

where $X:[0,1] \rightarrow \operatorname{conv}\left(R^{2}\right)$ is set-valued mapping, $P=\left\{x \in R^{2} \mid 0 \leq x_{1}-2 \leq 4,1 \leq\right.$ $\left.x_{2}-2 \leq 3\right\}$.

Each differential equation will have two basic solutions $X_{1}^{b g}(\cdot), X_{2}^{b g}(\cdot)$ and $X_{1}^{p s}(\cdot)$, $X_{2}^{p s}(\cdot)$ (see Figures 18,19 and Figures 20,21).

Example 3.11. Let

$$
\begin{aligned}
& D_{b g} X(t)=(-1) X(t), X(0)=P, t \in[0,1], \\
& D_{p s} X(t)=(-1) X(t), X(0)=P, t \in[0,1] .
\end{aligned}
$$

Also, each differential equation will have two basic solutions $X_{1}^{b g}(\cdot), X_{2}^{b g}(\cdot)$ and $X_{1}^{p s}(\cdot)$, $X_{2}^{p s}(\cdot)$ (see Figures 22, 23 and Figures 24, 25).

Remark 3.12. It's obvious that the basic solution $X_{2}^{p s}(\cdot)$ of differential equation (3.12) coincides with the basic solution $X_{2}^{b g}(\cdot)$ of differential equation (3.13). Also, the basic solution $X_{2}^{b g}(\cdot)$ of differential equation (3.11) coincides with the basic solution $X_{2}^{p s}(\cdot)$ 


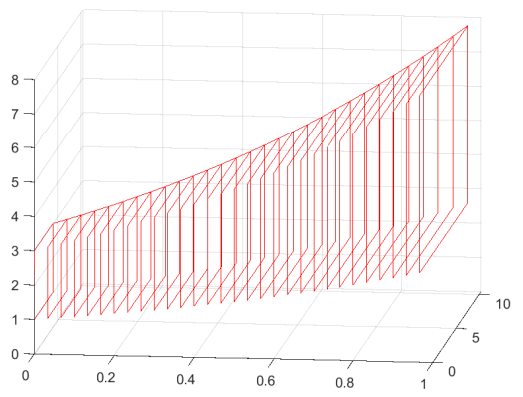

Figure 18: $X_{1}^{b g}(t), t \in[0,1]$

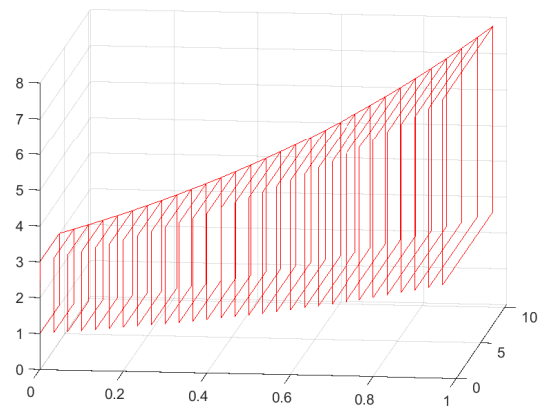

Figure 20: $X_{1}^{p s}(t), t \in[0,1]$

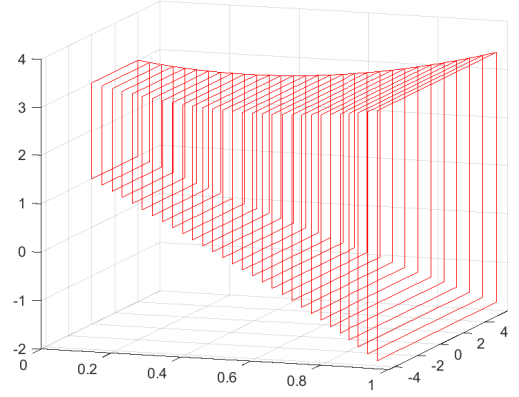

Figure 22: $X_{1}^{b g}(t), t \in[0,1]$

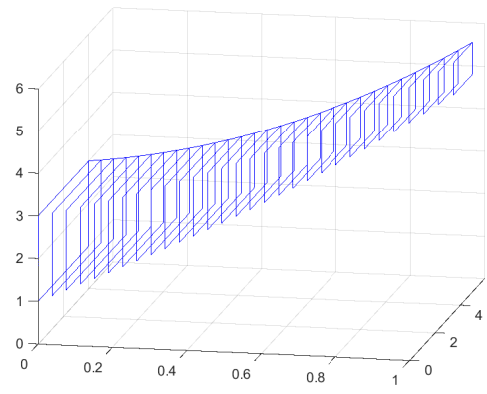

Figure 19: $X_{2}^{b g}(t), t \in[0,1]$

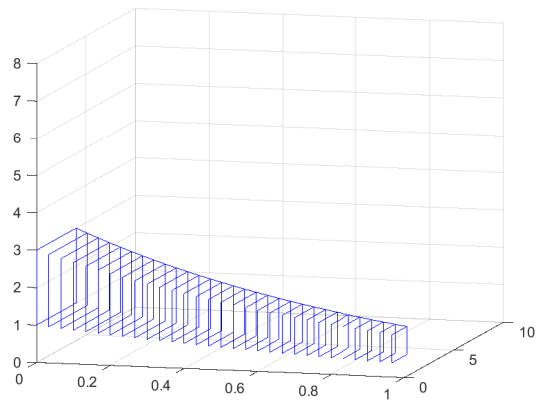

Figure 21: $X_{2}^{p s}(t), t \in[0,1]$

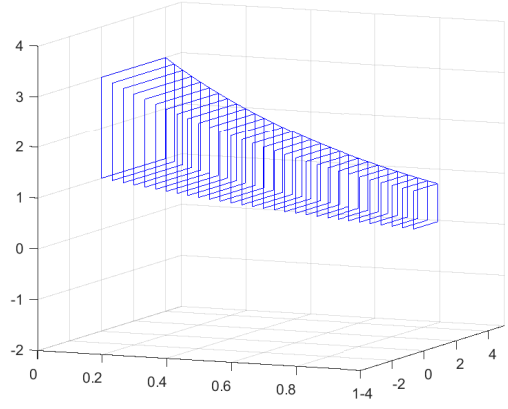

Figure 23: $X_{2}^{b g}(t), t \in[0,1]$

of differential equation (3.14). This is confirmed by integral equations that correspond to differential equations (3.11), (3.12), (3.13) and (3.14):

$$
X_{2}^{b g}(t)=P \frac{H}{(-1)} \int_{0}^{t} X_{2}^{b g}(s) d s,
$$




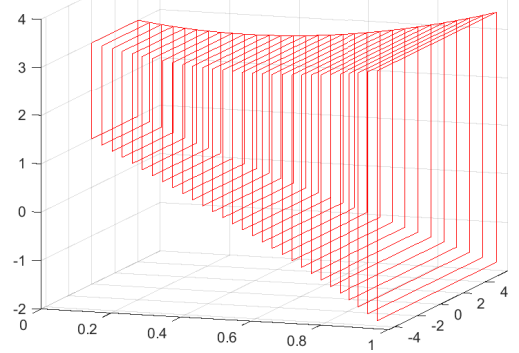

Figure 24: $X_{1}^{p s}(t), t \in[0,1]$

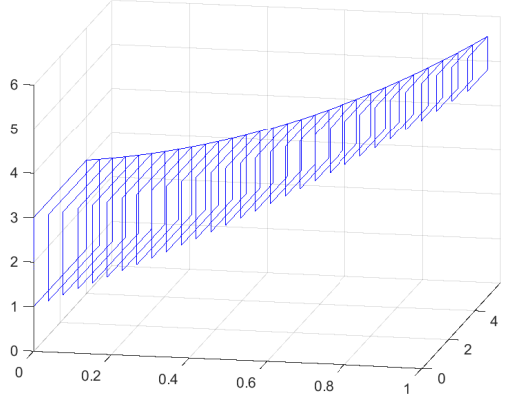

Figure 25: $X_{2}^{p s}(t), t \in[0,1]$

$$
\begin{gathered}
X_{2}^{p s}(t)=P \frac{H}{\int_{0}^{t}} X_{2}^{p s}(s) d s, \\
X_{2}^{b g}(t)=P \frac{H}{t}(-1) \int_{0}^{t}(-1) X_{2}^{b g}(s) d s=P \frac{H}{\int_{0}^{t}} X_{2}^{b g}(s) d s, \\
X_{2}^{p s}(t)=P \frac{H}{t} \int_{0}^{t}(-1) X_{2}^{p s}(s) d s=P \frac{H}{(-1)} \int_{0}^{p s} X_{2}^{p s}(s) d s .
\end{gathered}
$$

Remark 3.13. If the differential equation with the PS-derivative (BG-derivative) has two basic solutions and we write the corresponding system of interval-valued differential equations the PS-derivative (BG-derivative) similar to (3.3), then Remark 3.3 will be satisfied. However, we note that this system will always have two basic solutions (even when the original equation has only one basic solution).

Based on all above stated, we can make the following proposition.

Proposition 3.14. For system (3.1) the following statements are true:

1) if $H$-difference $X_{0} \underline{H}(-1) X_{0}$ exists, then differential equation (3.1) with $P S(B G)$-derivative has two basic solutions;

2) if $H$-difference $X_{0} \underline{H}(-1) X_{0}$ does not exist, then

a) if $a>0$, then differential equation (3.1) with PS-derivative has two basic solutions and differential equation (3.1) with BG-derivative has one basic solution;

a) if $a<0$, then differential equation (3.1) with BG-derivative has two basic solutions and differential equation (3.1) with PS-derivative has one basic solution.

\section{Conclusion}

In the article it is shown that linear set-valued differential equations have significant differences from ordinary and interval-valued linear differential equations. In these equations, the number of solutions may depend on the form (shape) of the initial 
set, the considered derivative and the coefficient in the right-hand side. We also note that in articles $[32,33,34,35,42]$, the authors considered a new type of differential equations with PS-derivative, in which no more than one solution can exist.

\section{References}

[1] Amrahov, Ş.E., Khastan, A., Gasilov, N., Fatullayev, A.G., Relationship between BedeGal differentiable set-valued functions and their associated support functions, Fuzzy Sets Syst., 265(2016), 57-72.

[2] Banks, H.T., Jacobs, M.Q., A differential calculus for multifunctions, J. Math. Anal. Appl., 29(1970), 246-272.

[3] Bede, B., Gal, S.G., Almost periodic fuzzy-number-valued functions, Fuzzy Sets Syst., 147(2004), 385-403.

[4] Bede, B., Gal, S.G., Generalizations of the differentiability of fuzzy number valued functions with applications to fuzzy differential equation, Fuzzy Sets Syst., 151 (2005), 581599.

[5] de Blasi, F.S., Iervolino, F., Equazioni differentiali con soluzioni a valore compatto convesso, Boll. Unione Mat. Ital., 2(4-5)(1969), 491-501.

[6] de Blasi, F.S., Lakshmikantham, V., Bhaskar, T.G., An existence theorem for set differential inclusions in a semilinear metric space, Control Cybernet., 36(3)(2007), 571-582.

[7] Boltyanski,V.G., Jerónimo Castro, J., Centrally symmetric convex sets, J. Convex Anal., 14(2)(2007), 345-351.

[8] Bridgland, T.F., Trajectory integrals of set valued functions, Pac. J. Math., 33(1)(1970), 43-68.

[9] Chalco-Cano, Y., Roman-Flores, H., Jimenez-Gamero, M.D., Generalized derivative and $\pi$-derivative for set-valued functions, Inform. Sci., 181(11)(2011), 2177-2188.

[10] Gomes, L.T., Barros, L., Bede, B., Fuzzy Differential Equations in Various Approaches, Springer Briefs in Mathematics, Springer, 2015.

[11] Hukuhara, M., Integration des applications mesurables dont la valeur est un compact convexe, Funkc. Ekvacioj, Ser. Int., 10(1967), 205-223.

[12] Komleva, T.A., Plotnikov, A.V., Differential inclusions with the Hukuhara derivative, Nonlinear Oscil., 10(2)(2007), 229-245.

[13] Komleva, T.A., Plotnikova, L.I., Plotnikov, A.V., Partial averaging of discrete-time setvalued systems, Stud. Univ. Babeş-Bolyai Math., 63(2018), no. 4, 539-548.

[14] Komleva, T.A., Plotnikova, L.I., Plotnikov, A.V., A multivalued discrete system and its properties, Ukr. Math. J., 70(11)(2019), 1750-1757.

[15] Komleva, T.A., Plotnikov, A.V., Skripnik, N.V., Differential equations with set-valued solutions, Ukr. Math. J., 60(10)(2008), 1540-1556.

[16] Lakshmikantham, V., Granna Bhaskar, T., Vasundhara Devi, J., Theory of Set Differential Equations in Metric Spaces, Cambridge Scientific Publishers, Cambridge, 2006.

[17] Lakshmikantham, V., Mohapatra, R.N., Theory of Fuzzy Differential Equations and Inclusions, Taylor \& Francis, London, 2003.

[18] Lasota, A., Strauss, A., Asymptotic behavior for differential equations which cannot be locally linearized, J. Differ. Equations, 10(1971), 152-172. 
[19] Malinowski, M.T., Second type Hukuhara differentiable solutions to the delay set-valued differential equations, Appl. Math. Comput., 218(2012), 9427-9437.

[20] Malinowski, M.T., On set differential equations in Banach spaces - a second type Hukuhara differentiability approach, Appl. Math. Comput., 219(2012), 289-305.

[21] Martelli, M., Vignoli, A., On differentiability of multi-valued maps, Boll. Unione Mat. Ital., 10(1974), 701-712.

[22] Perestyuk, N.A., Plotnikov, V.A., Samoilenko, A.M., Skripnik, N.V., Differential equations with impulse effects: multivalued right-hand sides with discontinuities, de Gruyter Stud. Math. 40, Berlin/Boston, Walter De Gruyter GmbH\& Co, 2011.

[23] Perestyuk, N.A., Skripnik, N.V., Averaging of set-valued impulsive systems, Ukr. Math. J., 65(2013), no. 1, 140-157.

[24] Perestyuk, N.A., Skripnik, N.V., Averaging of fuzzy systems, Ukr. Math. J., 70(2018), no. 3, 477-494.

[25] Petersen, I.R., Savkin, A.V., Robust Kalman Filtering for Signals and Systems with Large Uncertainties, Control Engineering, Birkhäuser, Boston, MA, 1999.

[26] Plotnikov, A.V., Averaging differential embeddings with Hukuhara derivative, Ukr. Math. J., 41(1989), no. 1, 112-115.

[27] Plotnikov, A.V., Controlled quasidifferential equations and some of their properties, Differ. Equ., 34(10)(1998), 1332-1336.

[28] Plotnikov, A.V., Differentiation of multivalued mappings. T-derivative, Ukr. Math. J., 52(8)(2000), 1282-1291.

[29] Plotnikov, A.V., Komleva, T.A., Molchanyuk, I.V., Existence and uniqueness theorem for set-valued Volterra-Hammerstein integral equations, Asian-European J. Math., 10(3)(2018), 12 pages.

[30] Plotnikov, V.A., Plotnikov, A.V., Vityuk, A.N., Differential Equations with a Multivalued Right-Hand side. Asymptotic Methods, AstroPrint, Odessa, 1999.

[31] Plotnikov, A.V., Skripnik, N.V., Differential Equations with "Clear" and Fuzzy Multivalued Right-Hand Side. Asymptotics Methods, AstroPrint, Odessa, 2009.

[32] Plotnikov, A.V., Skripnik, N.V., Set-Valued differential equations with generalized derivative, J. Adv. Res. Pure Math., 3(1)(2011), 144-160.

[33] Plotnikov, A., Skripnik, N., Existence and uniqueness theorems for generalized set differential equations, Int. J. Control Sc. Eng., 2(1)(2012), 1-6.

[34] Plotnikov, A.V., Skripnik, N.V., An existence and uniqueness theorem to the Cauchy problem for generalised set differential equations, Dyn. Contin. Discrete Impuls. Syst., Ser. A, Math. Anal., 20(4)(2013), 433-445.

[35] Plotnikov, A.V., Skripnik, N.V., Conditions for the existence of local solutions of setvalued differential equations with generalized derivative, Ukr. Math. J., 65(10)(2014), 1498-1513.

[36] Plotnikov, A.V., Skripnik, N.V., Existence and uniqueness theorem for set integral equations, J. Adv. Res. Dyn. Control Syst., 5(2)(2013), 65-72.

[37] Plotnikova, N.V., Systems of linear differential equations with p-derivative and linear differential inclusions, Sb. Math., 196(2005), 16771691.

[38] Plotnikova, N.V., Approximation of a bundle of solutions of linear differential inclusions, Nonlinear Oscill., 9(3)(2006), 375-390. 
[39] Polovinkin, E.S., Multivalued Analysis and Differential Inclusions, FIZMATLIT, Moscow, 2014.

[40] Radström, H., An embedding theorem for spaces of convex sets, Proc. Amer. Math. Soc., 3(1952), 165-169.

[41] Skripnik, N.V., Averaging of impulsive differential inclusions with Hukuhara derivative, Nonlinear Oscil., 10(3)(2007), 422-438.

[42] Skripnik, N.V., Three-step averaging scheme for set-valued differential equations with generalized derivative, J. Math. Sci., 236(3)(2019), 333-342.

[43] Stefanini, L., Bede, B., Generalized Hukuhara differentiability of interval-valued functions and interval differential equations, Nonlinear Anal., 71(2009), 13111328.

[44] Tolstonogov, A., Differential Inclusions in a Banach Space, Kluwer Academic Publishers, Dordrecht, 2000.

[45] Tyurin, Yu.N., Mathematical statement of the simplified model of industrial planning, Econ. Math. Meth., 3(1965), 391-409.

[46] Vu, H., Dong, L.S., Initial value problem for second-order random fuzzy differential equations, Adv. Difference Equ., 2015:373 (2015), 23 pages.

[47] Vu, H., Van Hoa, N., On impulsive fuzzy functional differential equations, Iran. J. Fuzzy Syst., 13(4)(2016), 79-94.

Tatyana A. Komleva

Department of Mathematics,

Odessa State Academy Civil Engineering and Architecture,

4, Didrihsona Street, 65029 Odessa, Ukraine

e-mail: t-komleva@ukr.net

Liliya I. Plotnikova

Department of Mathematics and Modeling Systems,

Odessa National Polytechnic University,

1, Shevchenko Avenue, 65044 Odessa, Ukraine

e-mail: liplotnikova@ukr.net

Natalia V. Skripnik

Department of Optimal Control and Economic Cybernetics,

Odessa National University after named I.I. Mechnikov,

2, Dvoryanskaya Street, 65082 Odessa, Ukraine

e-mail: natalia.skripnik@gmail.com

Andrej V. Plotnikov

Department of Information Technology and Applied Mathematics,

Odessa State Academy Civil Engineering and Architecture,

4, Didrihsona Street, 65029 Odessa, Ukraine

e-mail: a-plotnikov@ukr.net 